\title{
Rotación de antimicrobianos en \\ la Unidad de Terapia Intensiva: \\ ¿Es ésta una estrategia útil?
}

\author{
MARTÍN LASSO B.*
}

\section{Cycling of antimicrobial agents in the intensive care units: Is it a useful strategy?}

Antimicrobial resistance is a significant problem in the Intensive Care Unit related to increased morbidity, mortality and represents more costs in these facilities. Cycling of antibiotics is a new tool against bacterial resistance. In medical literature there is evidence of its benefits in lowering the emergence of resistant strains but clinical experience is still scarce and its implementation implies a profound change in guidelines and hospital policies that compromises the participation of all medical staff.

Key words: Cycling antibiotics; Intensive Care Unit; Antimicrobial resistance

\section{Introducción}

La presencia de resistencia antimicrobiana es un problema de gran importancia en las unidades de pacientes críticos, repercutiendo en costos de atención, morbilidad y mortalidad. Ha existido en los últimos años un incremento de patógenos resistentes en las infecciones nosocomiales de pacientes en UCI (Tabla 1). En nuestro país, Staphylococus aureus, Enterococcus spp, Klebsiella spp y Acinetobacter baumannii constituyen un problema creciente intrahospitalario de multiresistencia. La optimización en la aplicación de las medidas de control de infecciones como aislamiento, lavado de manos, uso de guantes y batas, y el correcto uso de antimicrobianos, son los puntales en el control de la emergencia y diseminación de patógenos resistentes.

La exposición previa y el uso indiscriminado de antimicrobianos son factores implicados en la aparición de resistencia; varias observaciones lo confirman: cambios en el uso de antimicrobianos se correlacionan con cambios en la prevalencia de resistencia, la resistencia antimicrobiana es más frecuente en cepas bacterianas nosocomiales que en las cepas provenientes de infecciones comunitarias, en los brotes de infecciones nosocomiales la contaminación o infección por agentes resistentes ocurre habitualmente en pacientes que han recibido antimicrobianos, las áreas

Tabla 1. Incidencia de cepas bacterianas resistentes en Unidades de Cuidados Intensivos en E.U.A. CDC/NNIS. Diciembre 2000

\begin{tabular}{llc}
\hline Microorganismo & Resistencia & Porcentaje (\%) \\
\hline Enterococcus sp & Vancomicina & 26,3 \\
Staphylococcus aureus & Meticilina & 55,3 \\
Staphylococcus coagulasa (-) & Meticilina & 87,5 \\
Escherichia coli & Cefalosporinas 3 & 3,4 \\
Klebsiella pneumoniae & Cefalosporinas 3 & gen \\
Pseudomonas aeruginosa & Imipenem & 11,2 \\
Pseudomonas aeruginosa & Quinolonas & 17,7 \\
Pseudomonas aeruginosa & Cefalosporina 3 & 27,3 \\
Enterobacter spp & Cefalosporinas 3 & 26,4 \\
& & 34,9 \\
\hline
\end{tabular}

\footnotetext{
* Servicio de Medicina. Unidad de Infectología. Hospital Dr Sótero Del Río.

E-mail:zoalde@yahoo.com
} 
hospitalarias que tienen mayor frecuencia de resistencia también son las que tienen mayor tasa de uso de antimicrobianos, la exposición prolongada a antimicrobianos se correlaciona con mayor posibilidad de colonización por agentes resistentes.

Los mecanismos por los cuales un antibacteriano lleva a la selección de microorganismos resistentes son varios: incremento y diseminación de cepas sensibles con mutaciones puntuales que se tornan resistentes por uso inadecuado de antimicrobianos, combinación de mutaciones entre cepas sensibles luego de un tratamiento inadecuado o exitoso, disminución de la resistencia a la colonización luego de tratamiento exitoso de una cepa sensible protectora que da paso, vía contaminación cruzada, a la colonización con cepas resistentes de otros pacientes, e incremento en la densidad de microorganismos resistentes en un mismo paciente luego de la eliminación de las especies sensibles. La genética de la resistencia subyace a estos fenómenos a través de varios mecanismos: la expresión de un gen de resistencia cromosomal latente o la adquisición de nuevo

Tabla 2. Principales estudios sobre rotación de antimicrobianos

\begin{tabular}{|c|c|c|c|c|}
\hline Autor * & $\mathbf{n}$ & Problema & Rotación & Resultado \\
\hline Gerding DN & Variable & BGN R** & $\begin{array}{c}\text { Amikacina, Gentamicina } \\
\text { y } \\
\text { tobramicina. }\end{array}$ & $\begin{array}{c}\text { Reducción de } \\
\text { BGN } \mathrm{R} \\
\text { a gentamicina } \mathrm{y} \\
\text { tobramicina } \\
\text { Incremento } \\
\text { P. aeruginosa } \mathrm{R} \text { a } \\
\text { amikacina }\end{array}$ \\
\hline Kollef MH & 3.668 & $\begin{array}{l}\text { Tratamiento } \\
\text { inadecuado de } \\
\text { infección } \\
\text { nosocomial }\end{array}$ & $\begin{array}{l}\text { Ceftazidima, } \\
\text { ciprofloxacina } \\
\text { y cefepime }\end{array}$ & $\begin{array}{c}\text { Disminuye uso } \\
\text { inadecuado y } \\
\text { mortalidad } \\
\text { de pacientes } \\
\text { con APACHE II > } 15 \\
\text { Disminuye BGN R a } \\
\text { cefalosporinas de } 3^{\circ} \text { gen }\end{array}$ \\
\hline Gruson D & 497 & $\begin{array}{c}\text { Alta tasa de NVM*** } \\
\text { por BGN R / } \\
P . \text { aeruginosa } \\
\text { en especial }\end{array}$ & $\begin{array}{l}\text { Ceftazidima }+ \\
\text { ciprofloxacina, } \\
\beta \text {-lactámicos, } \\
\text { y AMG**** }\end{array}$ & $\begin{array}{c}\text { Incremento de } \\
\text { susceptibilidad de } \\
P \text { aeruginosa } \\
\text { (de } 53,2 \text { a } 74,5 \%) \text {. } \\
\text { Disminución de NVM } \\
\text { asociados a BGN R } \\
\text { y mortalidad } \\
\text { sin cambios. }\end{array}$ \\
\hline Raymond DP & 540 & $\begin{array}{l}\text { Alta frecuencia } \\
\text { de BGN R. } \\
\text { Mortalidad elevada } \\
\text { en UCI quirúrgica }\end{array}$ & $\begin{array}{c}\text { Ciprofloxacina }+ \\
\text { clindamicina, } \\
\text { piperacilina/tazobactam, } \\
\text { carbapenems, } \\
\text { cefepime y } \\
\text { clindamicina }+ \\
\text { metronidazol }\end{array}$ & $\begin{array}{c}\text { Reducción de } \\
\text { incidencia de } \\
\text { infecciones por BGN R } \\
\text { y S aureus } \mathrm{R}\end{array}$ \\
\hline Moss WJ & 411 & $\begin{array}{l}\text { BGN R -2/3- } \\
\text { bacteriemias. } \\
\text { Estudio piloto } \\
\text { de seguridad }\end{array}$ & $\begin{array}{c}\text { Imipenem / cilastatina, } \\
\text { piperacilina/tazobactam } \\
\text { y ceftazidima, } \\
\text { y clindamicina }\end{array}$ & $\begin{array}{c}\text { Decrece colonización } \\
\text { y bacteriemia por } \\
\text { BGN R } \\
\text { No aparece } \\
\text { resistencia nueva }\end{array}$ \\
\hline
\end{tabular}

*Todas las fuentes constan en Referencias, **BGN R: bacilos Gram negativos resistentes, ***NVM: Neumonía en ventilación mecánica, ****AMG: aminoglucósidos. 
material de resistencia genética por intercambio directo de ADN, por bacteriófagos, plasmidios de ADN extracromosomales, transposones, integrones y gen casette, lo que lleva en último término a inactivación del antimicrobiano (vg: enzimas modificadoras de aminoglucosidos y $\beta$ lactamasas), modificación del sitio de acción o target del antibacteriano (PBP, ADN girasa y dehidrofolato reductasa) o que alteran su receptores bacterianos (enzimas que alteran la composición de la pared celular). En suma la presión selectiva antimicrobiana favorece la aparición de resistencia.

En las "Guías para la Prevención de Resistencia Antimicrobiana para Hospitales" (SHEA Position Paper. Infect Control Hosp Epidemiol 1997; 118 (4): 275-91) se proponen las bases para el adecuado manejo de antibacterianos en los hospitales, las cuales son:

- Uso óptimo de antimicrobianos.

- Remoción selectiva o restricción de antimicrobianos específicos o clases de antimicrobianos.

- Rotación de antimicrobianos.

- Uso de terapia combinada de antimicrobianos para prevenir la emergencia de resistencia.

La evidencia que soporta estas recomendaciones aún en la actualidad es limitada.

La rotación de antimicrobianos (RA) consiste en la sustitución programada de agentes antimicrobianos para el tratamiento de procesos infecciosos que ocurren en una unidad hospitalaria determinada a través de un ciclo preestablecido, por un período de tiempo definido, que implica el retorno al primer agente de la rotación y cuyo fin es disminuir la emergencia y diseminación de microorganismos resistentes a ellos, preservando la actividad de los antimicrobianos involucrados en el ciclo.

\section{Estudios clínicos de rotación de antimicrobianos}

La rotación de antimicrobianos no debe confundirse con la restricción de clase, esta última práctica es frecuentemente mencionada en la literatura como RA. El trabajo de Rahal et al (JAMA: 280 (14): 1233-7) realizado en un hospital de New York, es un ejemplo de restricción de clase, en él describe que, debido a la elevada frecuencia nosocomial de Klebsiella spp productoras de $\beta$-lactamasa de espectro extendido (BLEE) se decidió suprimir las cefalosporinas como antimicrobiano de elección durante un año, utilizando a cambio los carbapenémicos como "caballo de batalla" durante este período. El resultado fue que el hospital disminuyó el uso de cefalosporinas en $80 \%$ y la incidencia de Klebsiella resistente cayó en $44 \%$, (entre 70 y $87 \%$ en las unidades de intensivo); sin embargo, se registro un incremento de $140 \%$ en el uso de carbapenems acompañado de la emergencia de Pseudomonas resistente a imipenem la cual incremento su incidencia en $68 \%$. Al igual que en este, otros trabajos ponen en evidencia que la restricción de clase puede reducir la resistencia bacteriana al antimicrobiano suprimido pero tiene el riesgo de crear un nuevo problema de resistencia.

La RA representa una restricción de clases cíclica programada con aplicación de un esquema antimicrobiano definido y que retorna al uso del antibacteriano problema inicial en el supuesto que se ha recuperado la susceptibilidad de los microorganismos para ese agente.

Son pocos los trabajos referidos a RA (Tabla 2), y en ellos no se ha demostrado con claridad una disminución en la emergencia de resistencia antimicrobiana y la mayoría de ellos no cierra el ciclo para re-exponer al agente inicial. El trabajo clási-

Tabla 3. Uso y resistencia de aminoglucósidos entre bacilos Gram negativos durante cinco ciclos de rotación. Minneapolis Veteran`s Affairs Center: 1980 - 1990

\begin{tabular}{|c|c|c|c|c|c|c|}
\hline \multirow[b]{2}{*}{$\begin{array}{l}\text { Ciclo de } \\
\text { rotación }\end{array}$} & \multirow[b]{2}{*}{ Meses } & \multicolumn{2}{|c|}{ Uso Relativo (\%) } & \multirow{2}{*}{$\begin{array}{l}\text { Uso de AMG } \\
\text { (Pacientes } \\
\text { día/mes) }\end{array}$} & \multicolumn{2}{|c|}{ Porcentaje de resistencia } \\
\hline & & Amikacina & Gentamicina & & Amikacina & Gentamicina \\
\hline Basal & 3 & 1,2 & 76,6 & 496 & 3,8 & 12,0 \\
\hline Amikacina 1 & 26 & $92,3 *$ & $5,3 *$ & 705 & 3,2 & $6,4 *$ \\
\hline Gentamicina 1 & 12 & $31,5^{*}$ & $66,5^{*}$ & 777 & 3,9 & $9,2 *$ \\
\hline Amikacina 2 & 27 & $97,5^{*}$ & $0,9 *$ & 934 & 3,1 & $5,8 *$ \\
\hline Gentamicina 2 & 51 & $29,8^{*}$ & $68,2 *$ & 908 & 2,9 & 5,7 \\
\hline
\end{tabular}

$* \mathrm{p}<0,01$ comparado con período previo de estudio.

Modificado de Gerding DN Antimicrob Agents Chemother 1991; 35: 1284-90. 
Tabla 4. Rotación de antimicrobianos en una Terapia Intensiva Quirúrgica

\begin{tabular}{lll}
\hline Trimestre & \multicolumn{1}{c}{ Neumonía } & $\begin{array}{c}\text { Peritonitis o sepsis de origen } \\
\text { desconocido }\end{array}$ \\
\hline Enero/marzo & Ciprofloxacina +/- clindamicina* & Carbapenem \\
Abril/junio & Piperacilina/tazobactam & Cefepime + metronidazol** \\
Julio/septiembre & Carbapenem & $\begin{array}{l}\text { Ciprofloxacina }+ \text { clindamicina** } \\
\text { Piperacilina/tazobactam }\end{array}$ \\
\hline
\end{tabular}

*Indicada sólo ante sospecha de neumonía aspirativa, **Se agrega ampicilina o vancomicina si se sospecha Enterococcus spp.

Referencia 8.

co de Gerding DN et al, realizado por 10 años en el Minneapolis Veterans Affairs Medical Center (Tabla 3), es el punto de referencia de todos los trabajos relacionados con RA; en él se describe que, debido al incremento de la resistencia a gentamicina y tobramicina en Enterobacteriaceae (especialmente Kebsiella y Serratia), se decidió cambiar estos antimicrobianos por amikacina; con ello se logró un descenso marcado de la resistencia a gentamicina y tobramicina; con la re-introducción de gentamicina rápidamente se incremento el porcentaje de microorganismos resistentes lo que obligó a restituir la amikacina restringiendo nuevamente el uso de gentamicina; luego de 27 meses la gentamicina fue nuevamente introducida estabilizándose la resistencia en $5,7 \%$ lo que se consideró como una condición basal de resistencia "primaria" debido a que no se encontró plasmidios mediadores de resistencia a gentamicina en los microorganismos nosocomiales. Se estableció con este estudio que existe la posibilidad de frenar la emergencia de resistencia por medio de la RA.

La utilidad de esta práctica en la terapia intensiva aún no se ha definido. Raymond et al, en un estudio llevado acabo en la Universidad de Virginia entre 1997 y 1999, aplicó una rotación empírica de antimicrobianos entre pacientes postoperados de una UCI quirúrgica, considerando que este centro tenía un importante problema con $S$ aureus MR y bacterias Gram negativas productoras de BLEE; luego de un período previo de un año de observación se aplicó antimicrobianos empíricos, a dos brazos, a las intercurrencias infecciosas más frecuentes de esta población: neumonía y peritonitis o sepsis de origen desconocido. Se realizaron 4 ciclos en el transcurso de un año (Tabla 4). La adherencia a este programa fluctuó entre 62 y $83 \%$ y se demostró un beneficio respecto a la emergencia de resistencia antimicrobiana en el grupo sometido a rotación antimicrobiana (Tabla 5). A pesar de ello este

Tabla 5. Características de pacientes admitidos en UCI, incidencia de infección atribuida a la unidad y resistencia antimicrobiana

\begin{tabular}{lccc}
\hline & No Rotación & $\begin{array}{c}\text { Rotación } \\
\text { de antimicrobianos }\end{array}$ & P \\
\hline Admisiones en UCI & 699 & 757 & 0.77 \\
APACHE & $15,1 \pm 0,4$ & $15,2 \pm 0,4$ & \\
Infecciones nosocomiales & 175 & 142 & 0.0001 \\
Infecciones/100 admisiones & 42,3 & 32,2 & $<0.0001$ \\
Infecciones por Gram (+) MR & 102 & 59 & \\
Gram (+)MR/100 admisiones & 14,6 & 7,8 & $<0.0001$ \\
Infecciones por Gram (-) MR & 54 & 19 & 2,5 \\
Gram (-) MR/100 admisiones & 7,7 &
\end{tabular}

Adaptado de referencia 8. 
trabajo tiene el problema de que aquellos pacientes del grupo no sometido a rotación correspondían a infecciones tardías y era más frecuente la presencia de individuos que habían sido trasplantados, estaban en hemodiálisis o tenían daño hepático crónico que en el grupo en rotación; además junto con el inicio del período de RA se implementó el uso de un sistema de lavado en seco (alcohol gel) con la evidente influencia que esta medida tiene en disminuir la infección cruzada.

El más reciente trabajo sobre rotación de antimicrobianos lo realizó Moss et al en la UCI pediátrica del Johns Hopkins Hospital, y se generó debido a que más de dos tercios de las bacteriemias de esta unidad correspondían a bacilos Gram negativos resistentes. Se trató de un estudio piloto que tenía la finalidad de evaluar la seguridad del procedimiento de rotación y definir un tamaño de muestra que otorgara resultados significativos en un trabajo futuro. Luego de un período de 3 meses inicial basal, se decidió establecer una RA con la que se manejaría toda sospecha de infección que fuera admitida en la unidad, el orden fue el siguiente: imipenem/ cilastatina, piperacilina/tazobactam y ceftazidima + clindamicina, la terapia antimicrobiana era ajustada sólo ante la presencia de cultivos positivos significativos, cada ciclo duraba 3 meses y la RA se prolongó por 18 meses. Los resultados mostraron una disminución de la colonización por microorganismos resistentes de 34,9\% inicial a $25,8 \%$ luego de la RA, las bacteriemias nosocomiales también descendieron $27 \%$ en relación a otras UCI del mismo centro hospitalario, con excepción de una sola unidad que demostró un descenso igual y en la que se realizó una campaña de reforzamiento de las normas de control de infecciones concomitantemente al período de rotación de la UCI pediátrica. Este estudio concluyó que la RA es un procedimiento seguro, no origina nuevas cepas resistentes y tampoco favorece la aparición de micosis sistémica; además definió como significativo un descenso de $5 \%$ en la prevalencia de colonización por un agente resistente y señaló el tamaño de muestra de 1.460 pacientes para un poder de $80 \%$ y un nivel de significación de 5\%, lo que servirá para realizar un estudio futuro.

La interpretación de todos estos resultados debe pasar por el análisis de que en la terapia intensiva sólo 1 de cada 4 agentes aislados corresponde a una infección adquirida en UCI, de que los mecanismos de resistencia de los diversos patógenos son distintos y responden de diversa manera a la alteración de la presión antimicrobiana, que el análisis de los datos se basa en un antes y un después, careciendo de un grupo control contemporáneo y que no se considera en las discusiones la variación natural de la resistencia de los agentes infecciosos en el transcurso de los meses.

Por último, son especialmente interesantes la aplicación de modelos matemáticos fortuitos en estudios teóricos comparativos entre RA y el uso mixto de antimicrobianos. Lo $\mathrm{M}$ et al en un trabajo realizado en la Universidad de Harvard usó un modelo matemático fortuito de transmisión de bacterias susceptibles y resistentes y comparó varios esquemas de RA versus esquemas antimicrobianos mixtos en una UCI virtual de 20 camas, aplicó 240 parámetros diferentes relacionados a tipos de unidad, intensidad de transmisión, tránsito de pacientes, uso de antimicrobianos y capacidad de colonización bacteriana. Este trabajo demostró que en $77 \%$ de las situaciones (184/240) la carga de agentes colonizadores resistentes fue menor en el grupo de uso mixto de antimicrobianos con respecto al grupo de RA, estableciendo esta última práctica como poco eficiente en prevenir la resistencia.

\section{Recomendaciones para establecer una sistema de rotación de antimicrobianos}

Hay que entender que la meta de la RA no es sólo reducir la resistencia antimicrobiana del momento sino también prevenir la emergencia de nueva resistencia e influir en la morbi-mortalidad. Además, como se ha visto, no existe un trabajo adecuadamente estructurado que genere una validez de los resultados obtenidos; por ello es necesario establecer varios requisitos para montar un adecuado sistema de rotación de antimicrobianos:

- Precisar las definiciones de resistencia antimicrobiana.

- Establecer un sistema de monitoreo de la frecuencia de resistencia antimicrobiana.

- Capacidad de distinguir entre cepas bacterianas comunitarias y nosocomiales.

- Diferenciar con claridad si los agentes aislados representan infección o colonización.

- Capacidad de identificar aislamientos de acuerdo al sitio corporal y ubicación hospitalaria.

- Tener un estable y efectivo sistema de control de infecciones hospitalarias.

- Sistema que asegure el apropiado uso de todos los antimicrobianos con un mecanismo claro de entrega de los mismos, idealmente con apoyo computacional.

- Establecer un plan de elección para definir 
qué antimicrobianos o clase de antimicrobianos pueden entrar en la rotación.

- Planificar la duración de cada ciclo y el orden del mismo. Ciclos de rotación trimestrales en 18 meses parece ser más conveniente, pero ello debería ajustarse a los determinantes de resistencia de los microorganismos problema. Ciclos muy largos se asocian a desarrollo de resistencia.

- Debe existir un período previo a la R A en el que se mida dirigidamente la frecuencia de infecciones nosocomiales, la presencia de microorganismos multiresistentes, mortalidad y costos, aplicando las normas establecidas de control de antimicrobianos y precauciones universales y específicas del centro en estudio.

- Estrategia para controlar y reforzar las rotaciones.

- Capacidad de controlar y monitorizar los mecanismos de resistencia y su genética.

- Capacidad de tipificar los microorganismos y establecer clonalidad en base a la genética.

- Un método para medir el uso de antimicrobianos por área geográfica y por unidad de tiempo.

- Monitoreo de evolución clínica para lograr que el paciente adhiera a las políticas de la rotación.

- Apoyo de los médicos de la unidad para la realización del programa ya que $\sim 10$ a $50 \%$ de pacientes dentro de un programa de rotación reciben antimicrobianos "fuera de ciclo" debido a consideraciones clínicas como alergias, efectos adversos o aplicación de guías locales o nacionales.

- No establecer una presión de vigilancia excesiva entre investigadores y tratantes.

- Establecer un plan para realizar un estudio que genere resultados válidos: definir significación clínica y un grupo control contemporáneo.

- Se debe seleccionar los pacientes a quienes se puede aplicar una RA, excluyendo aquellos en los cuales el beneficio de un determinado antimicrobiano empírico es absolutamente claro como en los casos de meningitis bacteriana aguda o la peritonitis bacteriana espontánea, o protocolos ya establecidos, como los episodios de neutropenias febriles o la endocarditis bacteriana.

- Establecer como política del hospital la RA instituyendo una programación obligatoria y evitando la adherencia voluntaria la que se ha visto relacionada a fracaso en estos protocolos.

En suma la RA puede resultar un procedimiento interesante para frenar la resistencia bacteriana, pero su real utilidad aún no se ha demostrado y es necesario disponer de nuevos estudios especialmente locales para definir su beneficio.

\section{Resumen}

La resistencia antimicrobiana es un problema muy relevante en las unidades de terapia intensiva y se relaciona directamente con la mayor morbilidad, mortalidad y elevados costos que se generan en estas unidades. La rotación de antimicrobianos es una nueva herramienta en el combate de la resistencia. Existe evidencia en la literatura de su beneficio en la disminución de la emergencia de microorganismos resistentes pero la experiencia clínica no es suficiente aún y su aplicación implica todo un cambio en las normas y políticas hospitalarias obligando además a la participación comprometida de todo el estamento médico.

\section{Bibliografía}

1.- Fridkin S K. Routine cycling of antimicrobial agents as an infection-control measure. Clin Infect Dis 2003; 36: $1438-44$.

2.- Gerding D N. Antimicrobial cycling: Lessons learned from the aminoglycoside experience. Infect Control Hosp Epidemiol. 2000 Jan; 21(1 Suppl): S12-S7.

3.- Gruson D, Hilbert G, Vargas F, Valentino R, Bebear C, Allery A et al. Rotation and restricted use of antibiotics in a Medical Intensive Unit. Am J Respir Crit Care Med 2000; 162: 837-43.

4.- John J F Jr, Rice L B. The microbial genetics of antibiotic cycling. Infect Control Hosp Epidemiol. 2000 Jan; 21 (1 Suppl): S22-S31.

5.- Kollef M H, Ward S, Sherman G et al. Inadequate treatment of nosocomial infections is associated with certain empiric antibiotic choices. Crit Care Med; 2000: 28 (10): 3456-64.

6.- Pujol M, Gudiol F. Evidence for antibiotic cycling in control of resistance. Curr Opin Infect Dis 2001; 14 (6): 711-5.

7.- Moss W J, Beers M C, Johnson E et al. Pilot study of antibiotic cycling in a pediatric intensive care unit. Crit Care Med 2002; 30 (8): 1877-82.

8.- Raymond D P, Pelletier S J, Crabtree T D et al. Impact of a rotating empiric antibiotic schedule on infectious mortality in an intensive care unit. Crit Care Med. 2001; 29 (6): 1101-8.

9.- Weinstein R A. Controlling antimicrobial resistance in hospitals: Infection control and use of antibiotics. Emerg Infect Dis 2001; 7 (2): 188-92. 